\title{
Global Solutions for a Simplified Shallow Elastic Fluids Model
}

\author{
Yun-guang Lu, ${ }^{1}$ Christian Klingenberg, ${ }^{2}$ Leonardo Rendon, ${ }^{3}$ and De-Yin Zheng ${ }^{1}$ \\ ${ }^{1}$ Department of Mathematics, Hangzhou Normal University, Hangzhou 310036, China \\ ${ }^{2}$ Institute of Mathematics, University of Wurzburg, Emil Fischer Straße 30, 97074 Wurzburg, Germany \\ ${ }^{3}$ Departamento de Matemáticas, Universidad Nacional de Colombia, Bogotá, Colombia
}

Correspondence should be addressed to De-Yin Zheng; deyinzheng@hzno.edo.cn

Received 22 September 2013; Accepted 10 February 2014; Published 19 March 2014

Academic Editor: Adem Kilicman

Copyright (C) 2014 Yun-guang Lu et al. This is an open access article distributed under the Creative Commons Attribution License, which permits unrestricted use, distribution, and reproduction in any medium, provided the original work is properly cited.

The Cauchy problem for a simplified shallow elastic fluids model, one $3 \times 3$ system of Temple's type, is studied and a global weak solution is obtained by using the compensated compactness theorem coupled with the total variation estimates on the first and third Riemann invariants, where the second Riemann invariant is singular near the zero layer depth $(\rho=0)$. This work extends in some sense the previous works, (Serre, 1987) and (Leveque and Temple, 1985), which provided the global existence of weak solutions for $2 \times 2$ strictly hyperbolic system and (Heibig, 1994) for $n \times n$ strictly hyperbolic system with smooth Riemann invariants.

\section{Introduction}

In [1], the authors proposed a new reduced model for gravity-driven free-surface flows of shallow elastic fluids. It is obtained by an asymptotic expansion of the upper-convected Maxwell model for elastic fluids. The viscosity is assumed small, but the relaxation time is kept finite. The simplified system of (5.6) in [1] is as follows:

$$
\begin{gathered}
\rho_{t}+(\rho u)_{x}=0, \\
(\rho u)_{t}+\left(\rho u^{2}+\pi\right)_{x}=0, \\
\left(\rho \frac{\pi}{s^{2}}\right)_{t}+\left(\rho u \frac{\pi}{s^{2}}+u\right)_{x}=0, \\
s_{t}+u s_{x}=0, \\
c_{t}+u c_{x}=0 .
\end{gathered}
$$

Since the variable $c$ in the last equation is independent of the first four equations in (1), we remove it and let $v=\pi / s^{2}, s=$ const. $>0$ to obtain the following conservation system:

$$
\begin{gathered}
\rho_{t}+(\rho u)_{x}=0, \\
(\rho u)_{t}+\left(\rho u^{2}+s^{2} v\right)_{x}=0, \\
(\rho v)_{t}+(\rho u v+u)_{x}=0 .
\end{gathered}
$$

By simple calculations, three eigenvalues of system (2) are

$$
\lambda_{1}=u-\frac{s}{\rho}, \quad \lambda_{2}=u, \quad \lambda_{1}=u-\frac{s}{\rho}
$$

with corresponding Riemann invariants

$$
R_{1}=s^{2} v-s u, \quad R_{2}=v+\frac{1}{\rho}, \quad R_{3}=s^{2} v+s u,
$$

and all characteristic fields are of the Temple type, namely, the shock waves and rarefaction waves coincide.

In this paper, we will study the Cauchy problem of system (2) with bounded initial data

$$
\begin{array}{r}
(\rho(x, 0), u(x, 0), v(x, 0))=\left(\rho_{0}(x), u_{0}(x), v_{0}(x)\right), \\
\rho_{0}(x) \geq 0
\end{array}
$$

and prove the existence of global weak solutions by using the vanishing viscosity method coupled with the compensated compactness argument.

The hyperbolic systems of two conservation laws whose shock and rarefaction curves coincide were first described by Temple in [2], and later the global existence of weak solutions for such systems, with any bounded variation initial condition, were proven by Serre in [3] (also [4] for a special system), where the key technique is that the total variation of 
the Riemann invariants is decreasing in time. Serre's idea was extended by Heibig [5] to study any $n \times n$ strictly hyperbolic system of Temple type, with smooth flux functions. For system (2), both the flux functions and the second Riemann invariant $R_{2}$ are singular near the zero layer depth $\rho=0$. So, the technique proposed in $[3,5]$ cannot be used to study system (2) directly. Fortunately, we may accept the method in [3] to obtain that the total variation, of the first and third Riemann invariants, is decreasing in time, which implies the $H_{\text {loc }}^{-1}$ compactness of $u_{x}^{\varepsilon}$ and $v_{x}^{\varepsilon}$ for the viscosity solutions $\left(u^{\varepsilon}, v^{\varepsilon}\right)$ given by the Cauchy problem. Then we may apply for the div-curl lemma to the pairs of functions $\left(c, u^{\varepsilon}\right)$ and $\left(c, v^{\varepsilon}\right)$, where $c$ is a constant, to give a very short proof of the existence of global weak solutions for the Cauchy problem (2) and (5).

Theorem 1. Suppose (I)

$$
\begin{gathered}
c_{1} \leq u_{0}(x)-s v_{0}(x) \leq c_{2}, \quad c_{3} \leq u_{0}(x)+s v_{0}(x) \leq c_{4}, \\
\frac{1}{\rho_{0}(x)+\varepsilon}+v_{0}(x)>c_{5},
\end{gathered}
$$

where $\varepsilon$ is a small positive constant and $c_{i}, i=1,2, \ldots, 5$ are suitable constants satisfying

$$
c_{5}-\frac{c_{4}-c_{1}}{2 s}>0
$$

(II) the total variations of $u_{0}(x)-s v_{0}(x)$ and $u_{0}(x)+s v_{0}(x)$ are bounded, then the Cauchy problem (2) and (5) has a bounded measurable solution ( $\rho, u, v), \rho \geq 0$ satisfying system (2) in the sense of distributions.

The existing result given in [5] is based on that for any system of Temple type; there exists a strictly convex entropy (Lemma 1, [5]). However, in our case, the existence of a strictly convex entropy is not obvious since all pairs of entropy-entropy flux of (2) are constructed in the following theorem.

Theorem 2. All the entropies of (2) are in the form

$$
\eta(\rho, u, v)=\rho\left(F(u+s v)+G(u-s v)+H\left(v+\frac{1}{\rho}\right)\right),
$$

where $F, G$, and $H$ are arbitrary functions, with corresponding entropy flux

$$
\begin{gathered}
q(\rho, u, v)=(\rho u+s)(F(u+s v)+(\rho u-s) G(u-s v) \\
\left.+\rho u H\left(v+\frac{1}{\rho}\right)\right) .
\end{gathered}
$$

Furthermore when $F, G$, and $H$ are all convex, the entropy is convex.

We will prove Theorems 1 and 2 in Sections 2 and 3, respectively.

\section{Proof of Theorem 1}

We will prove Theorem 1 by several lemmas.

Consider the Cauchy problem for the related parabolic system

$$
\begin{gathered}
\rho_{t}+(\rho u)_{x}=\varepsilon \rho_{x x}, \\
(\rho u)_{t}+\left(\rho u^{2}+s^{2} v\right)_{x}=\varepsilon(\rho u)_{x x} \\
(\rho v)_{t}+(\rho u v+u)_{x}=\varepsilon(\rho v)_{x x}
\end{gathered}
$$

with initial data

$$
\begin{aligned}
& \left(\rho^{\varepsilon}(x, 0), u^{\varepsilon}(x, 0), v^{\varepsilon}(x, 0)\right) \\
& \quad=\left(\rho_{0}^{\varepsilon}(x), u_{0}^{\varepsilon}(x), v_{0}^{\varepsilon}(x)\right) \\
& \quad=\left(\rho_{0}(x)+\varepsilon, u_{0}(x), v_{0}(x)\right) * G^{\varepsilon},
\end{aligned}
$$

where $G^{\varepsilon}$ is a mollifier.

Lemma 3. If the initial data satisfy (6) and (7), then for any fixed $\varepsilon>0$, the viscosity solution $\left(\rho^{\varepsilon}, u^{\varepsilon}, v^{\varepsilon}\right)$ of the Cauchy problem (10) and (11) exists.

Proof of Lemma 3. We substitute the first equation into the second and third equations in (10), respectively, to obtain

$$
\begin{array}{r}
R_{i t}\left(\rho^{\varepsilon}, u^{\varepsilon}, v^{\varepsilon}\right)+\lambda_{i} R_{i x}\left(\rho^{\varepsilon}, u^{\varepsilon}, v^{\varepsilon}\right) \\
=\varepsilon R_{i x x}\left(\rho^{\varepsilon}, u^{\varepsilon}, v^{\varepsilon}\right)+\frac{2 \varepsilon}{\rho} \rho_{x} R_{i x}\left(\rho^{\varepsilon}, u^{\varepsilon}, v^{\varepsilon}\right), \\
i=1,2,3 .
\end{array}
$$

Then by applying the maximum principle to (12), we have

$$
\begin{gathered}
c_{1} \leq R_{1}\left(\rho^{\varepsilon}, u^{\varepsilon}, v^{\varepsilon}\right) \leq c_{2}, \quad c_{3} \leq R_{3}\left(\rho^{\varepsilon}, u^{\varepsilon}, v^{\varepsilon}\right) \leq c_{4}, \\
R_{2}\left(\rho^{\varepsilon}, u^{\varepsilon}, v^{\varepsilon}\right)>c_{5},
\end{gathered}
$$

due to the initial conditions given in (6), where $R_{i}$ are Riemann invariants given in (4).

Using these estimates, we have the following bounded estimates on $\left(\rho^{\varepsilon}, u^{\varepsilon}, v^{\varepsilon}\right)$ :

$$
\begin{gathered}
\frac{c_{3}-c_{2}}{2 s} \leq v^{\varepsilon} \leq \\
\frac{c_{4}-c_{1}}{2 s}, \quad \frac{c_{1}+c_{3}}{2} \leq u^{\varepsilon} \leq \frac{c_{2}+c_{4}}{2}, \\
\frac{1}{\rho^{\varepsilon}}>c_{5}-\frac{c_{4}-c_{1}}{2 s}>0
\end{gathered}
$$

due to the condition in (7). Since the boundedness of $u^{\varepsilon}$, we have the positive, lower estimate

$$
\rho^{\varepsilon}(x, t) \geq c(t, \varepsilon)>0,
$$

by using Theorem 1.0.2 in [6] and the first equation in (10), where $c(t, \varepsilon)$ could tend to zero as the time $t$ tends to infinity or $\varepsilon$ tends to zero.

The standard theory of semilinear parabolic systems, namely, the local existence and the a priori bounded estimates in (14) and (15), gives us the existence of global smooth solution for the Cauchy problem (10) and (11). Lemma 3 is proven. 
Lemma 4. If the total variations of $u_{0}(x)-s v_{0}(x)$ and $u_{0}(x)+$ $s v_{0}(x)$ are bounded, then the viscosity solutions $\left(\rho^{\varepsilon}, u^{\varepsilon}, v^{\varepsilon}\right)$ satisfy

$$
\begin{aligned}
& \int_{-\infty}^{\infty}\left|u_{x}^{\varepsilon}(x, t)-s v_{x}^{\varepsilon}(x, t)\right| d x \leq M, \\
& \int_{-\infty}^{\infty}\left|u_{x}^{\varepsilon}(x, t)+s v_{x}^{\varepsilon}(x, t)\right| d x \leq M,
\end{aligned}
$$

where $M$ is a positive constant, which is independent of $\varepsilon$.

Proof of Lemma 4. Let $\phi=R_{1 x}$. Choose $i=1$ in (12). Differentiating (12) with respect to $x$ and then multiplying the sequence of smooth functions $h^{\prime}(\phi, \alpha)$ to the result, we have

$$
\begin{gathered}
\phi_{t}+\left(\lambda_{1} \phi\right)_{x}=\varepsilon \phi_{x x}+\left(2 \varepsilon \rho^{-1} \rho_{x} \phi\right)_{x} \\
h(\phi, \alpha)_{t}+\left(\lambda_{1} h(\phi, \alpha)\right)_{x}+\left(h^{\prime}(\phi, \alpha) \phi-h(\phi, \alpha)\right) \lambda_{1 x} \\
=\varepsilon h(\phi, \alpha)_{x x}-\varepsilon h^{\prime \prime}(\phi, \alpha) \phi_{x}^{2}+\left(2 \varepsilon \rho^{-1} \rho_{x} h(\phi, \alpha)\right)_{x} \\
+\left(2 \varepsilon \rho^{-1} \rho_{x}\right)_{x}\left(h^{\prime}(\phi, \alpha) \phi-h(\phi, \alpha)\right) .
\end{gathered}
$$

If we choose a convex $h(\phi, \alpha), h^{\prime \prime}(\phi, \alpha) \geq 0$ and $h^{\prime}(\phi, \alpha) \rightarrow$ $\operatorname{sign} \phi, h(\phi, \alpha) \rightarrow|\phi|$ as $\alpha \rightarrow 0$, then we let $\alpha \rightarrow 0$ in (17) and we have

$$
|\phi|_{t}+\left(\lambda_{1}|\phi|\right)_{x} \leq \varepsilon|\phi|_{x x}+\left(2 \varepsilon \rho^{-1} \rho_{x}|\phi|\right)_{x}
$$

in the sense of distributions. Integrating (18) in $R \times[0, t]$, we have

$$
\begin{aligned}
\int_{-\infty}^{\infty}\left|R_{1 x}\right|(x, t) d x & =\int_{-\infty}^{\infty}|\phi|(x, t) d x \\
& \leq \int_{-\infty}^{\infty}|\phi|(x, 0) d x \leq M
\end{aligned}
$$

since $T V R_{10}(x)$ is bounded. So, we proved the first estimate in (16). Similarly we can prove the second estimate in (16). Lemma 4 is proven.

\section{Lemma 5.}

$$
\begin{array}{ll}
f\left(u^{\varepsilon}(x, t)\right)_{x}, & g\left(v^{\varepsilon}(x, t)\right)_{x} \\
\text { are compact in } & H_{\mathrm{loc}}^{-1}\left(R \times R^{+}\right),
\end{array}
$$

where $f(u), g(v)$ are any smooth functions.

Proof of Lemma 5. Using (16) and (14), we know that $f\left(u^{\varepsilon}(x, t)\right)_{x}$ and $g\left(v^{\varepsilon}(x, t)\right)_{x}$ are uniformly bounded both in $L_{\text {loc }}^{1}\left(R \times R^{+}\right)$and in $W_{\text {loc }}^{-1, \infty}\left(R \times R^{+}\right)$and so compact in $H_{\text {loc }}^{-1}\left(R \times R^{+}\right)$due to Murat's lemma [7]. Lemma 5 is proven.

\section{Lemma 6.}

$$
\begin{gathered}
\rho_{t}^{\varepsilon}+\left(\rho^{\varepsilon} u^{\varepsilon}\right)_{x}, \quad\left(\rho^{\varepsilon} u^{\varepsilon}\right)_{t}+\left(\rho^{\varepsilon}\left(u^{\varepsilon}\right)^{2}+s^{2} v^{\varepsilon}\right)_{x} \\
\left(\rho^{\varepsilon} v^{\varepsilon}\right)_{t}+\left(\rho^{\varepsilon} u^{\varepsilon} v^{\varepsilon}+u^{\varepsilon}\right)_{x}
\end{gathered}
$$

are compact in $H_{\mathrm{loc}}^{-1}\left(R \times R^{+}\right)$.
Proof of Lemma 6. Multiplying $l \rho^{l-1}(l>0)$ to the first equation in (10) (for simplicity, we omit the superscript $\varepsilon$ ), we have

$$
\rho_{t}^{l}+\left(\rho^{l} u\right)_{x}+(l-1) \rho^{l} u_{x}=\varepsilon \rho_{x x}^{l}-\varepsilon l(l-1) \rho^{l-2} \rho_{x}^{2} .
$$

Since $u_{x}$ is bounded in $L_{\text {loc }}^{1}\left(R \times R^{+}\right)$, multiplying a suitable nonnegative test function to $(22)$, we have that

$$
\varepsilon \rho^{l-2} \rho_{x}^{2} \text { is bounded in } L_{\text {loc }}^{1}\left(R \times R^{+}\right) .
$$

So the right-hand side of the first equation in (10) is compact in $H_{\mathrm{loc}}^{-1}\left(R \times R^{+}\right)$, which implies the $H_{\mathrm{loc}}^{-1}\left(R \times R^{+}\right)$compactness of $\rho_{t}^{\varepsilon}+\left(\rho^{\varepsilon} u^{\varepsilon}\right)_{x}$.

We substitute the first equation into the second in (10) to obtain

$$
u_{t}+u u_{x}+\frac{s^{2}}{\rho} v_{x}=\varepsilon u_{x x}+\frac{2 \varepsilon}{\rho} \rho_{x} u_{x} .
$$

Then we multiply (24) by $\rho h^{\prime}(u)$, the first equation in (10) by $h(u)$, then add the result to obtain

$$
\begin{gathered}
(\rho h(u))_{t}+(\rho u h(u))_{x}+s^{2} h^{\prime}(u) v_{x} \\
=\varepsilon(\rho h(u))_{x x}-\varepsilon h^{\prime \prime}(u) \rho u_{x}^{2} .
\end{gathered}
$$

Choosing a strictly convex function $h(u)$ and multiplying a suitable nonnegative test function to (25), we have that

$$
\varepsilon \rho u_{x}^{2} \text { is bounded in } L_{\text {loc }}^{1}\left(R \times R^{+}\right) .
$$

Similarly we can prove that

$$
\varepsilon \rho v_{x}^{2} \text { is bounded in } L_{\text {loc }}^{1}\left(R \times R^{+}\right) .
$$

Using (23), (26), (27), and (20), we may prove the $H_{\mathrm{loc}}^{-1}(R \times$ $R^{+}$) compactness of the last two terms in (21). Lemma 6 is proven.

Now we give the proof of Theorem 1 . Let $\left(\rho, u, v, \overline{h\left(\rho, u^{\varepsilon}, v^{\varepsilon}\right)}\right)$ be the weak star limits of $\left(\rho^{\varepsilon}, u^{\varepsilon}, v^{\varepsilon}, h\left(\rho^{\varepsilon}, u^{\varepsilon}, v^{\varepsilon}\right)\right)$.

Using the div-curl lemma $[6,8]$ to the following pairs of functions, respectively:

$$
\begin{gathered}
\left(c, u^{\varepsilon}\right), \quad\left(\rho^{\varepsilon}, \rho^{\varepsilon} u^{\varepsilon}\right) ; \\
\left(c, u^{\varepsilon}\right), \quad\left(\rho^{\varepsilon} u^{\varepsilon}, \rho^{\varepsilon}\left(u^{\varepsilon}\right)^{2}+s^{2} v^{\varepsilon}\right) ; \\
\left(c, v^{\varepsilon}\right), \quad\left(\rho^{\varepsilon}, \rho^{\varepsilon} u^{\varepsilon}\right) ; \\
\left(c, v^{\varepsilon}\right), \quad\left(\rho^{\varepsilon} u^{\varepsilon}, \rho^{\varepsilon}\left(u^{\varepsilon}\right)^{2}+s^{2} v^{\varepsilon}\right),
\end{gathered}
$$

where $c$ is a constant, we have

$$
\begin{array}{ll}
\overline{\rho^{\varepsilon} u^{\varepsilon}}=\rho u, & \overline{\rho^{\varepsilon}\left(u^{\varepsilon}\right)^{2}}=\overline{\rho^{\varepsilon} u^{\varepsilon}} \cdot \overline{u^{\varepsilon}}=\rho u^{2}, \\
\overline{\rho^{\varepsilon} v^{\varepsilon}}=\rho v, & \overline{\rho^{\varepsilon} v^{\varepsilon} u^{\varepsilon}}=\overline{\rho^{\varepsilon} u^{\varepsilon}} \cdot \overline{v^{\varepsilon}}=\rho u v .
\end{array}
$$

Therefore we may prove that the limit $(\rho, u, v)$ satisfies $(2)$ in the sense of distributions by letting $\varepsilon$ go to zero in (10). Theorem 1 is proven. 


\section{Proof of Theorem 2}

In this section, we will prove Theorem 2 . It is easy to prove that any pair of entropy-entropy flux $(\eta(\rho, u, v), q(\rho, u, v))$, of system (2), must satisfy the following system:

$$
\begin{gathered}
q_{\rho}=u \eta_{\rho}, \\
q_{u}=\rho \eta_{\rho}+u \eta_{u}+\frac{1}{\rho} \eta_{v}, \\
q_{v}=\frac{s^{2}}{\rho} \eta_{u}+u \eta_{v} .
\end{gathered}
$$

Then we have

$$
\begin{gathered}
q_{\rho u}=\eta_{\rho}+u \eta_{\rho u}=q_{u \rho}=\eta_{\rho}+\rho \eta_{\rho \rho} \\
+u \eta_{\rho u}-\frac{1}{\rho^{2}} \eta_{v}+\frac{1}{\rho} \eta_{v \rho}, \\
q_{\rho v}=u \eta_{\rho v}=q_{v \rho}=-\frac{s^{2}}{\rho^{2}} \eta_{u}+\frac{s^{2}}{\rho} \eta_{u \rho}+u \eta_{\rho v}, \\
q_{u v}=\rho \eta_{\rho v}+u \eta_{u v}+\frac{1}{\rho} \eta_{v v} \\
=q_{v u}=\frac{s^{2}}{\rho} \eta_{u u}+\eta_{v}+u \eta_{v u},
\end{gathered}
$$

which is

$$
\begin{gathered}
\rho^{3} \eta_{\rho \rho}+\rho \eta_{v \rho}=\eta_{v}, \\
\rho \eta_{u \rho}=\eta_{u}, \\
\rho^{2} \eta_{\rho v}+\eta_{v v}=s^{2} \eta_{u u}+\rho \eta_{v} .
\end{gathered}
$$

Using the first and third equations in (32), we obtain

$$
\eta_{v v}-\rho^{4} \eta_{\rho \rho}=s^{2} \eta_{u u}
$$

Resolving the second equation in (32), we have

$$
\eta_{u}=\rho h_{1}(u, v)
$$

where $h_{1}$ is an arbitrary function of $(u, v)$.

We rewrite the first equation in (32) as

$$
\left(\frac{\eta_{v}}{\rho}\right)_{\rho}=-\rho \eta_{\rho \rho}
$$

Then

$$
\frac{\eta_{v}}{\rho}=-\rho \eta_{\rho}+\eta+h_{2}(u, v) \text { or } \eta_{v}=\rho\left(\eta-\rho \eta_{\rho}\right)+\rho h_{2}(u, v),
$$

where $h_{2}$ is an arbitrary function of $(u, v)$.

Differentiating (36) with respect to $v$ and using the first equation in (32), we have

$$
\eta_{v v}=\rho\left(\eta_{v}-\rho \eta_{v \rho}\right)+\rho h_{2 v}(u, v)=\rho^{4} \eta_{\rho \rho}+\rho h_{2 v}(u, v) \text {. }
$$

Differentiating (34) with respect to $u$ and using (33) and (37), we have

$$
h_{2 v}(u, v)=s^{2} h_{1 u}(u, v) .
$$

Moreover, differentiating (34) with respect to $v$ and differentiating (36) with respect to $u$ and using the second equation in (32), we have

$$
h_{2 u}(u, v)=h_{1 v}(u, v) .
$$

We resolve (38) and (39) to obtain

$$
\begin{gathered}
h_{1}(u, v)=f(u+s v)+g(u-s v), \\
h_{2}(u, v)=s f(u+s v)-s g(u-s v),
\end{gathered}
$$

where $f, g$ are two arbitrary functions.

Integrating (34) with respect to $u$, we have

$$
\eta(\rho, u, v)=\rho(F(u+s v)+G(u-s v))+h(\rho, v),
$$

where $F^{\prime}=f, G^{\prime}=g$, and $h$ is a suitable function of $(\rho, v)$ such that $\eta$ given in (41) must satisfy (36). Thus $h$ satisfies

$$
h_{v}(\rho, v)=\rho h(\rho, v)-\rho^{2} h_{\rho}(\rho, v) \text { or }\left(\frac{h}{\rho}\right)_{v}+\rho^{2}\left(\frac{h}{\rho}\right)_{\rho}
$$

and so $h / \rho=H(v+1 / \rho)$ or $h(\rho, v)=\rho H(v+1 / \rho)$. Thus (8) is proven.

To prove (9), we remove all the terms on the right-hand side of (12) to obtain

$$
\begin{gathered}
(u-s v)_{t}+\left(u-\frac{s}{\rho}\right)(u-s v)_{x}=0 \\
\left(\frac{1}{\rho}+v\right)_{t}+u\left(\frac{1}{\rho}+v\right)_{x}=0 \\
(u+s v)_{t}+\left(u+\frac{s}{\rho}\right)(u+s v)_{x}=0 .
\end{gathered}
$$

Multiplying the first equation in (43) by $\rho G^{\prime}(u-s v)$ and the first equation in $(2)$ by $G(u-s v)$, then adding the result, we have

$$
(\rho G)_{t}+((\rho u-s) G)_{x}=0 .
$$

Thus the entropy flux corresponding to the entropy $\rho G(u-s v)$ is $q_{1}=(\rho u-s) G(u-s v)$.

Similarly, we may prove that the entropy flux corresponding to the entropy $\rho F(u+s v)$ is $q_{2}=(\rho u+s) F(u+s v)$, the flux corresponding to the entropy $\rho H(v+1 / \rho)$ is $q_{3}=$ $\rho u H(v+1 / \rho)$. Thus (9) is proven.

Let $\eta=\rho F(u+s v), m=\rho u$ and $n=\rho v$. By simple calculations

$$
\begin{gathered}
\eta_{\rho \rho}=\frac{1}{\rho}\left(\frac{m+s n}{\rho}\right)^{2} F^{\prime \prime}, \quad \eta_{m m}=\frac{1}{\rho} F^{\prime \prime}, \\
\eta_{n n}=\frac{s^{2}}{\rho} F^{\prime \prime}, \quad \eta_{\rho m}=-\frac{m+s n}{\rho^{2}} F^{\prime \prime}, \\
\eta_{\rho n}=-s \frac{m+s n}{\rho^{2}} F^{\prime \prime}, \quad \eta_{m n}=\frac{s}{\rho} F^{\prime \prime}
\end{gathered}
$$


and for any vector $(a, b, c)$

$$
\begin{gathered}
\eta_{\rho \rho} a^{2}+\eta_{m m} b^{2}+\eta_{n n} c^{2}+2 \eta_{\rho m} a b+2 \eta_{\rho n} a c+2 \eta_{m n} b c \\
=\frac{1}{\rho} F^{\prime \prime}\left(\frac{m+s n}{\rho} a-b-s c\right)^{2} \geq 0, \text { as } F^{\prime \prime} \geq 0 .
\end{gathered}
$$

Thus $\eta=\rho F(u+s v)$ is convex when $F$ is convex. Similarly we can prove both $\rho G(u-s v)$ and $\rho H(v+1 / \rho)$ are convex when $G, H$ are convex. Theorem 2 is proven.

\section{Conflict of Interests}

The authors declare that there is no conflict of interests regarding the publication of this paper.

\section{Acknowledgments}

This work was partially supported by the Natural Science Foundation of Zhejiang Province of China (Grant no. LY12A01030 and no. LZ13A010002) and the National Natural Science Foundation of China (Grant no. 11271105).

\section{References}

[1] F. Bouchut and S. Boyaval, "A new model for shallow elastic uids," preprint, 2011, http://www.math.ntnu.no/conservation/.

[2] B. Temple, "Systems of conservation laws with invariant submanifolds," Transactions of the American Mathematical Society, vol. 280, no. 2, pp. 781-795, 1983.

[3] D. Serre, "Solutions à variations bornées pour certains systèmes hyperboliques de lois de conservation," Journal of Differential Equations, vol. 68, no. 2, pp. 137-168, 1987.

[4] R. J. LeVeque and B. Temple, "Stability of Godunov's method for a class of $2 \times 2$ systems of conservation laws," Transactions of the American Mathematical Society, vol. 288, no. 1, pp. 115-123, 1985.

[5] A. Heibig, "Existence and uniqueness of solutions for some hyperbolic systems of conservation laws," Archive for Rational Mechanics and Analysis, vol. 126, no. 1, pp. 79-101, 1994.

[6] Y. G. Lu, Hyperbolic Conservation Laws and the Compensated Compactness Method, vol. 128, Chapman and Hall, CRC Press, New York, NY, USA, 2002.

[7] F. Murat, "Compacité par compensation," Annali della Scuola Normale Superiore di Pisa, vol. 5, no. 3, pp. 489-507, 1978.

[8] T. Tartar, "Compensated compactness and applications to partial differential equations," in Nonlinear Analysis and Mechanics: Heriot-Watt Symposium, R. J. Knops, Ed., vol. 4 of Research Notes in Mathematics, pp. 2797-2815, Pitman Press, London, UK, 1979. 


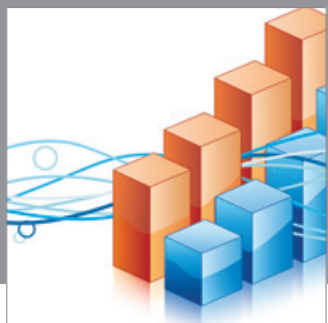

Advances in

Operations Research

mansans

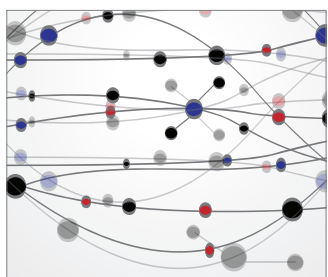

The Scientific World Journal
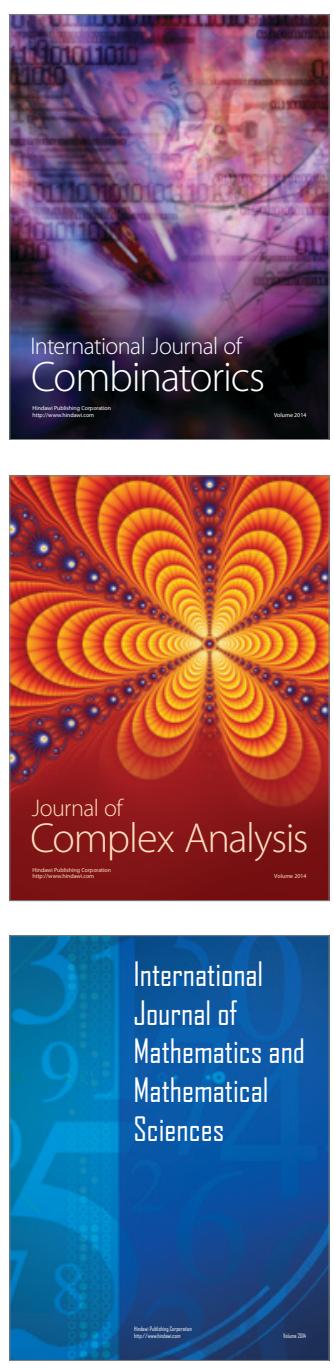
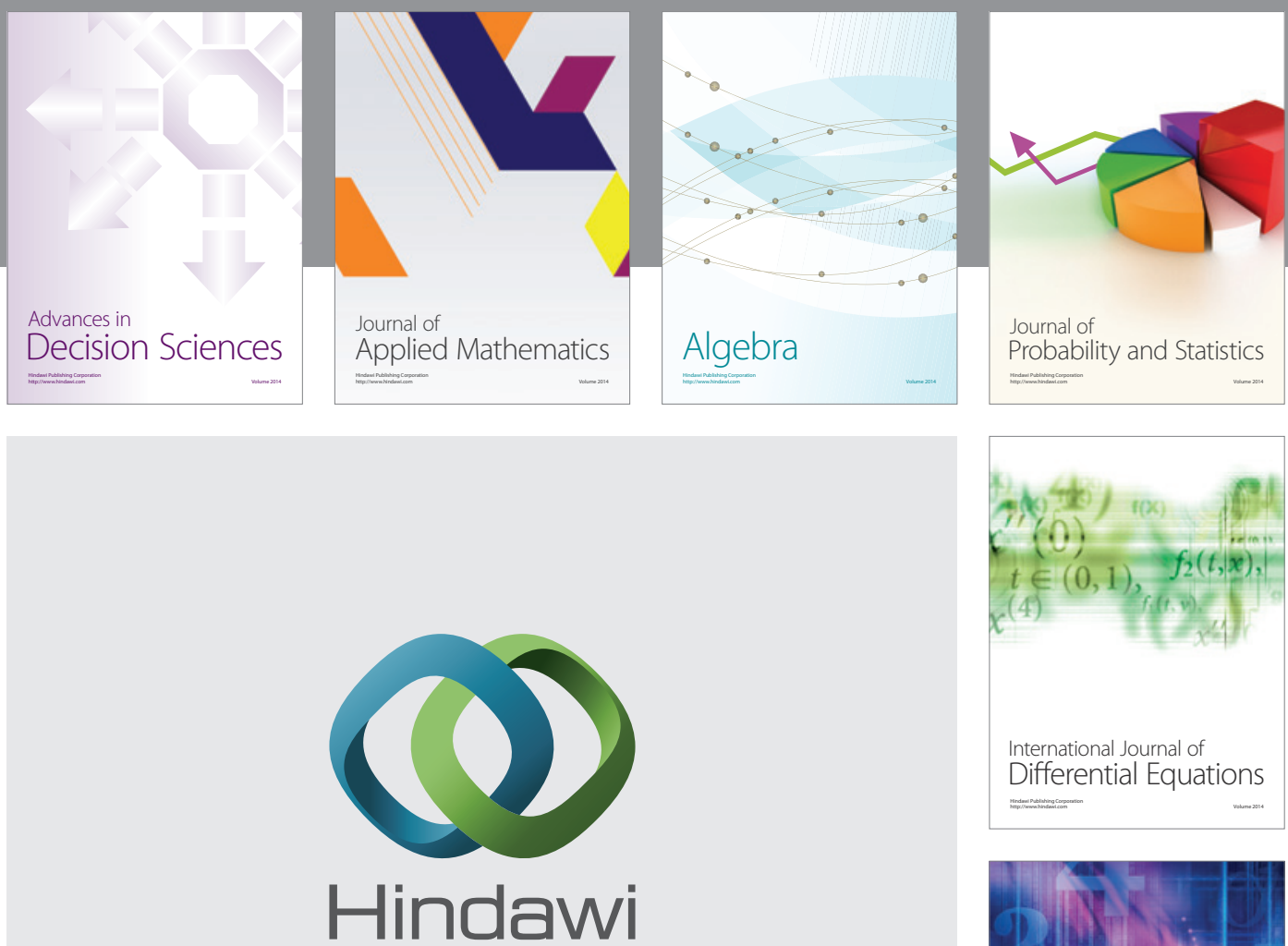

Submit your manuscripts at http://www.hindawi.com
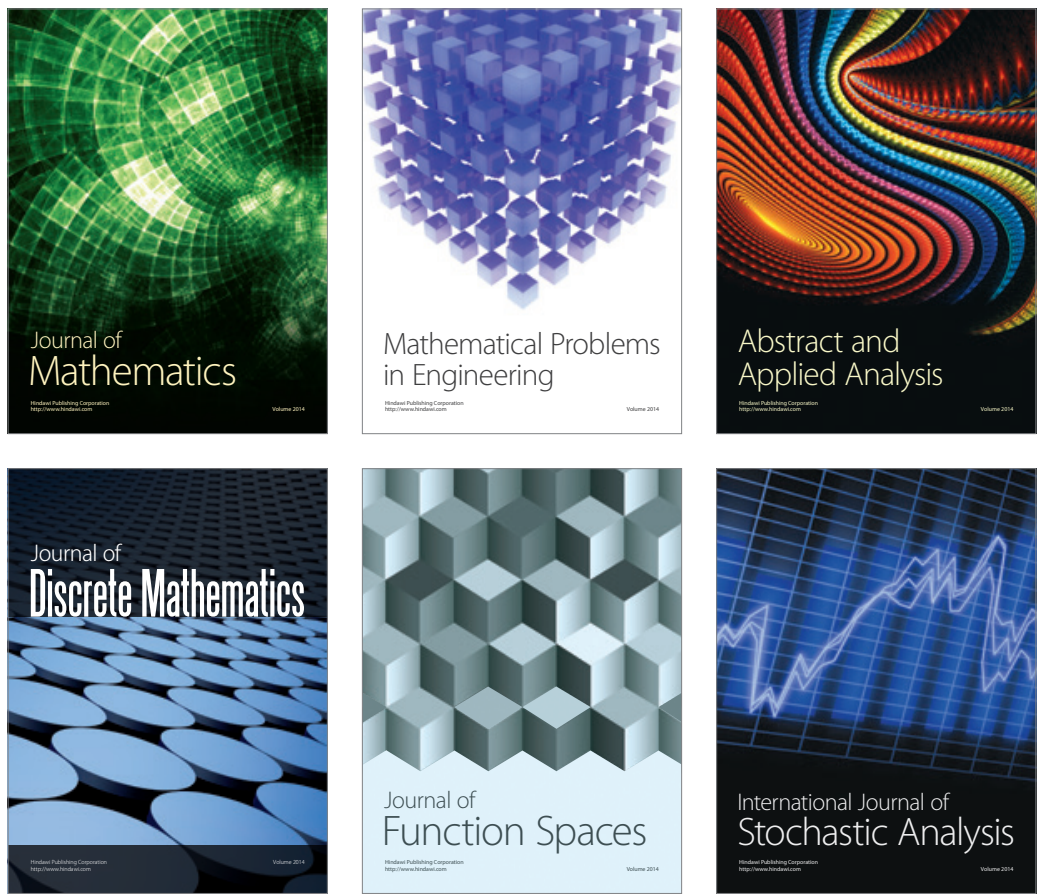

Journal of

Function Spaces

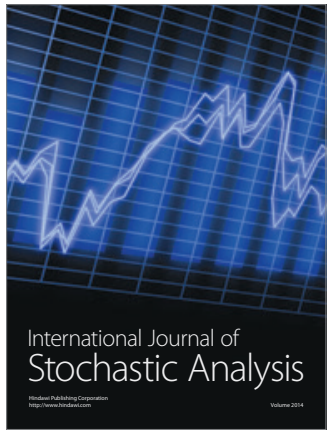

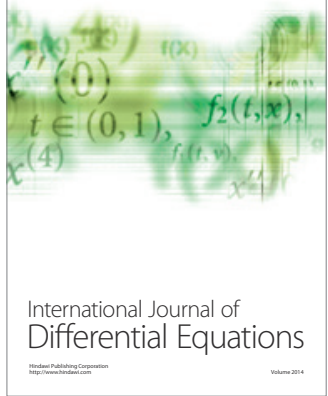
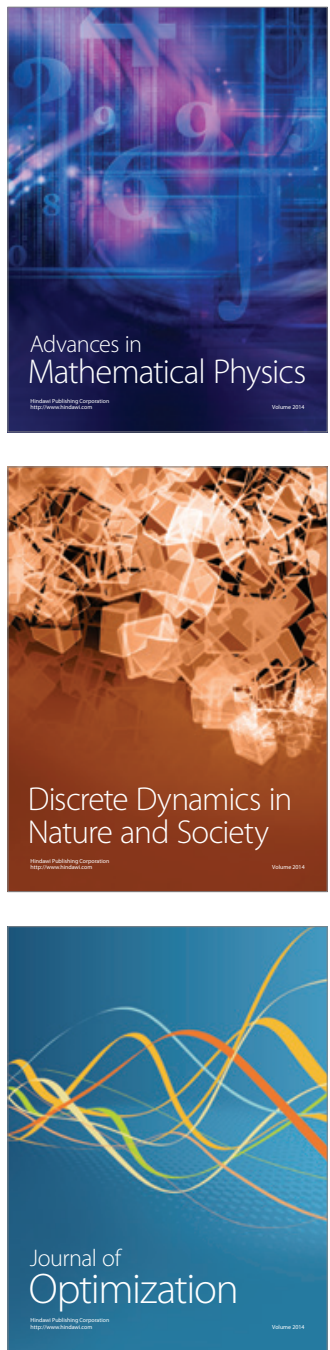